\title{
Spectral Tuning of Chlorophylls in Proteins - Electrostatics vs. Ring Deformation
}

\author{
Yigal Lahav ${ }^{\mathrm{a}, \mathrm{b}}$, Dror Noy*b and Igor Schapiro*a \\ aFritz Haber Center for Molecular Dynamics Research, Institute of Chemistry, , Jerusalem 9190401, \\ Israel.

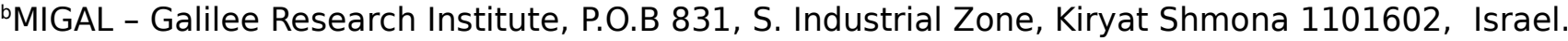

KEYWORDS: Chlorophyll binding proteins, Spectral Tuning, QM/MM, Molecular Dynamics.

\begin{abstract}
In photosynthetic complexes, tuning of chlorophyll light-absorption spectra by the protein environment is crucial to their efficiency and robustness. Water Soluble Chlorophyll-binding Proteins from Brassicaceae (WSCPs) are useful for studying spectral tuning mechanisms due to their symmetric homotetramer structure, the ability to rigorously modify the chlorophyll's protein surroundings, and the availability of crystal structures. Here, we present a rigorous analysis based on hybrid Quantum Mechanics and Molecular Mechanics simulations with conformational sampling to quantify the relative contributions of steric and electrostatic factors to the absorption spectra of WSCP-chlorophyll complexes. We show that when considering conformational dynamics, chlorophyll ring deformation accounts for about one-third of the spectral shift, whereas protein electrostatics accounts for the remaining twothirds. From a practical perspective, protein electrostatics is easier to manipulate than chlorophyll conformations, thus, it may be more readily implemented in designing artificial protein-chlorophyll complexes with desired spectral shift.
\end{abstract}

Photosynthetic organisms utilize elaborate transmembrane multi-subunit protein complexes, known as photosystems, to carry out the primary processes of solar energy conversion. The photosystems assemble and organize hundreds of pigments, all interacting with the protein environment and with one another. ${ }^{1,2}$ These interactions serve to tune the spectral properties of individual pigments, particularly their ground and excited states energy levels, for highly efficient light capture, energy transfer, and charge separation ${ }^{3,4}$.

Understanding the molecular mechanisms of this spectral tuning is very challenging even though the molecular structures of the photosystems are known with unprecedented detail, and to near atomic resolution ${ }^{1}$. This is mainly because the asymmetric arrangement of the pigments, and the small variations in their site energies result in highly congested optical spectra. Determining individual excitation energies from such convoluted spectra and assigning them to specific pigments can only be accomplished computationally. Yet, calculating the optical spectra of such asymmetric multipigment arrays is computationally very demanding, and cannot be achieved using quantum chemical methods without introducing additional approximations ${ }^{5-12}$. Simpler, smaller and more symmetric protein-pigment complexes may be useful experimental models for identifying and understanding how colour tuning mechanisms work. In addition, they may provide benchmarks for evaluating the validity of computational methods.

Such a promising model and benchmark is the class of type II Water Soluble Chlorophyll-binding Proteins (WSCPs) from Brassicaceae. ${ }^{13-18}$ These bind chlorophylls (Chls) with very high-affinity at 1:1 protein:pigment stoichiometry, and assemble them in homotetrameric complexes. Threedimensional structures of two complexes, namely, WSCPS from cauliflower (CaWSCP, PDB ID: 5HPZ) ${ }^{19}$ and Virginia pepperweed (LvWSCP, PDB ID: $2 \mathrm{DRE})^{20}$, are available at high-resolution, which is particularly suitable for computational modelling. The structures are representative of the type IIa, and type IIb, subclasses of type II WSCPs, respectively, that are distinguished by the energies of their Chls' $Q_{y}$ absorption band. The Chl a complex of the type lla absorbs at $\lambda_{\max }$ $=673 \mathrm{~nm}(1.842 \mathrm{eV})$, whereas the type Ilb 
complex absorbs at $\lambda_{\max }=664 \mathrm{~nm}(1.867 \mathrm{eV})$. Both structures reveal the Chls organized in a similar D2 symmetric configuration indicating that the spectral shifts are due to proteinpigment interactions.

An important advantage of using type II WSCPs for studying spectral tuning is the ability to rigorously modify the protein environment of the Chls by assembling them in vitro with recombinantly expressed proteins ${ }^{21-24}$. By implementing this strategy, Bednarczyk et al. ${ }^{19}$ demonstrated a spectral tuning mechanism based on a single-point mutation accounting for two-thirds of the spectral shifts between CaWSCP, and LvWSCP. Comparison of the crystal structures revealed a change in the position of a tryptophan residue (W151, or W154 in CaWSCP, or LVWSCP, respectively) in the near vicinity of the bound Chl (Fig. 1 a-b).

This displacement is caused by a change in the tryptophan hydrogen bonding to an amino acid located two residues downstream of the Chl's central magnesium coordination site - a proline backbone oxygen. In CaWSCP, a hydrogen bond between the $\varepsilon 1$ nitrogen of $\mathrm{W} 151$ and the backbone oxygen of an alanine (A34) brings W151 close to the Chl ring A (Fig. 1a), whereas in LvWSCP, the bond is shifted to the $\delta 1$ oxygen of an asparagine (N38) that replaces the alanine at the equivalent position and pushes W154 away from the Chl (Fig. 1b). Indeed, the A34N mutation in CaWSCP resulted in a blue shift of the Chl a Qy band from 673 to $667 \mathrm{~nm}$, while the N38A mutation in LvWSCP resulted in a red shift from 664 to $669 \mathrm{~nm}$. Based on these results Bednarczyk et al. ${ }^{19}$ assigned the primary cause of the spectral tuning to be the Chl ring deformation induced by the nearby tryptophan in CaWSCP which is relaxed in LvWSCP.

Ring deformation has long been considered a significant factor for inducing red shifts in the absorption spectra of porphyrin and chlorin derivatives ${ }^{25,26}$, and it becomes apparent when comparing the Chl conformations in the CaWSCP and LvWSCP structures (Fig. 1c, d). Recently, De Vico et al. proposed, based on quantum chemical calculations, that ring deformations of bacteriochlorophylls are the main reason for a $30 \mathrm{~nm}$ shift in the Qy absorption band of LH2 and LH3, two homologues of the light harvesting system from the purple bacteria Rhodoblastus acidophilus. ${ }^{27}$

To further quantify the role of ring deformation in tuning Chl spectra, we set out to explore the variations of protein and Chl molecular and electronic structure among the two WSCP complexes. To this end, we used the hybrid Quantum Mechanics/Molecular Mechanics (QM/MM) approach to determine the relative contributions to the observed shifts of CaWSCP- vs. LvWSCP-bound Chl spectra. The symmetry of the quaternary structure, and the similar relative orientations of the Chl macrocycles in both WSCPs allow us to perform the quantum calculations only on a single Chl under the reasonable assumption that all four binding sites are identical, and the Chls' excitonic coupling ${ }^{28,29}$ effects are similar.
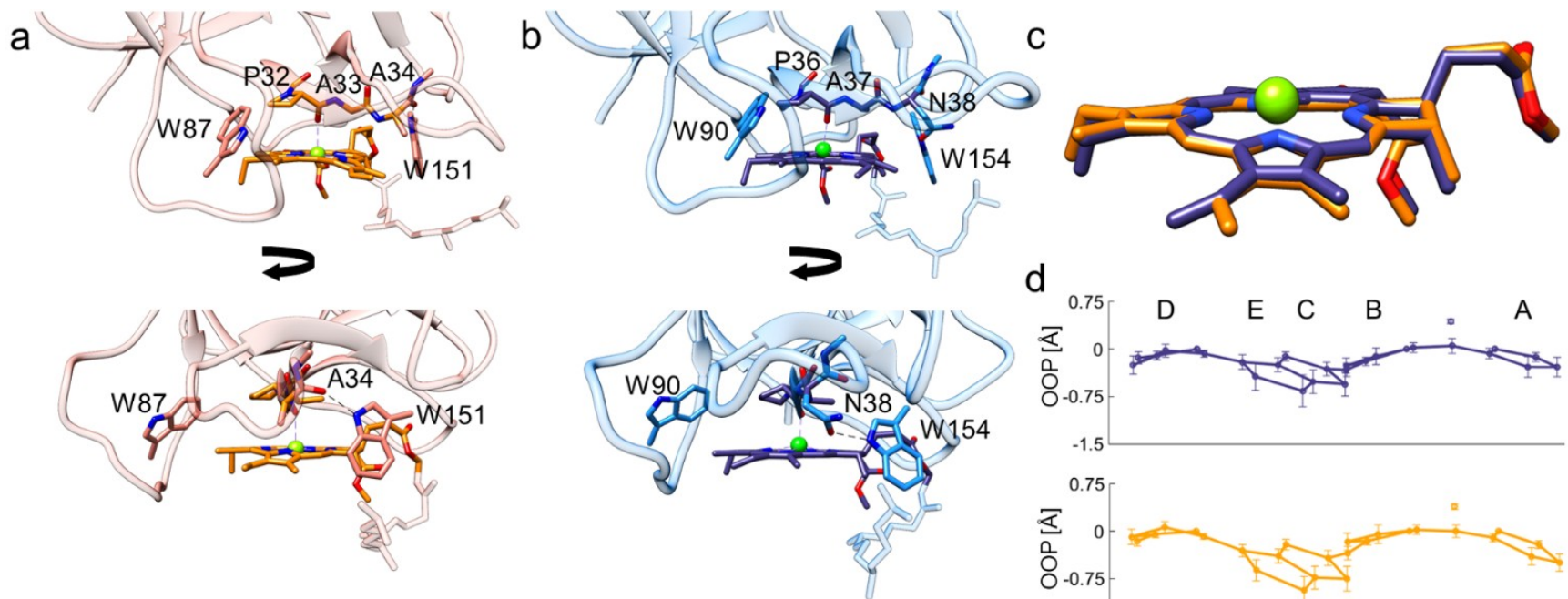

d
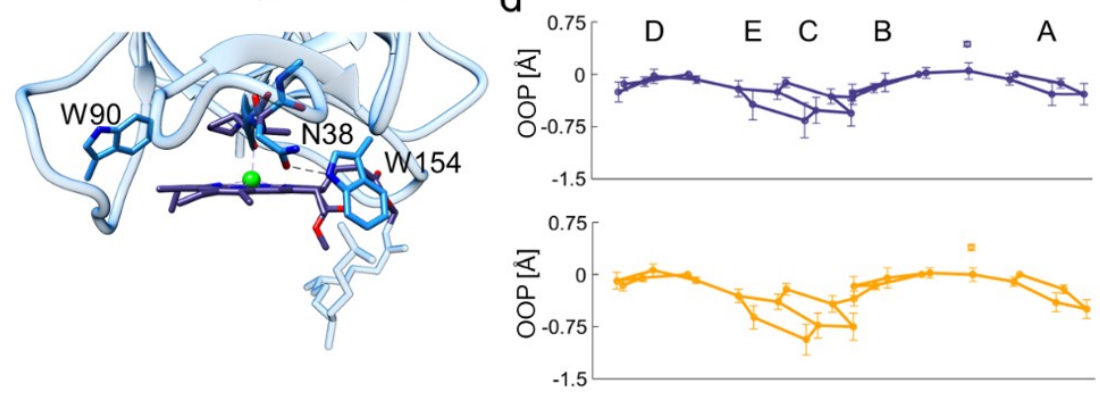

Figure 1. Chl binding sites and macrocycle conformations in CaWSCP and LvWSCP. Protein structures are shown in semi-transparent ribbon representation. Chls are in stick representation whereby atoms treated at the QM level are opaque, and the phytyl chain atoms treated at the MM level are semi-transparent. Dashed lines mark coordinative bonds to the Chl's central Mg atom (green sphere), and hydrogen bond to tryptophan $\varepsilon 1$ nitrogen. a) CaWSCP (PDB ID: 5HPZ). Residues P32, A33 and the Chl without phytyl chain (orange) were treated at the DFT level during the QM/MM relaxation. Residues A34, W151 and W87 (pink) were also included in the QM region for excitation energy calculations. b) LvWSCP (PDB ID: 2DRE), Residues P36, A37 and the Chl (purple) without phytyl chain were treated at the DFT level during the QM/MM relaxation. Residues N38, W154 and W90 (blue) were also included in the QM region for excitation 
energy calculation. c) 3D structure of LvWSCP (purple) and CaWSCP (orange), averaged over 100, QM/MM relaxed, snapshots. d) Out of Plane (OOP) displacement of the averaged Chl geometry in LvWSCP (top, purple) and CaWSCP (bottom, orange). Bars represent the root mean square deviation of each atom.

Our aim was to separate and quantify different contributions such as the effect of protein-induced electrostatics on excitation energies on one hand, and steric factors on the other hand. To this end, we established a computational setup that could reliably describe the variations of Chl absorption spectra in the different WSCP complexes. Then, we were able to quantify the effect of steric and electrostatic factors on the calculated $\mathrm{Chl}$ spectra by virtually turning these factors "on" or "off" in the model.

To calculate reliable absorption spectra, it was necessary to obtain information about the dynamics of the molecular system beyond the single geometry provided by the crystal structures of the WSCP complexes. ${ }^{30}$ This is because the size and complexity of these protein-pigment systems prohibit the use of a single vertical excitation energy at an equilibrium geometry (VEQ) that is commonly used for small organic molecules. A prerequisite for VEQ is the optimization of a minimum on the potential energy surface that can be identified and subsequently used for the calculation of vertical excitation energy. However, determining the absolute minimum of the potential energy surface in a protein is virtually impossible due to the high number of degrees of freedom and the associated presence of multiple minima. Here, we addressed the issue by sampling molecular conformations from molecular dynamics (MD) simulations and then performing a QM/MM relaxation on each sampled geometry (snapshot) at the density functional theory (DFT) level. The ensemble of these molecular conformations was used for calculating excitation energies at the time-dependent DFT (TD-DFT) level of theory. Recently, such an approach was demonstrated to provide a good description of absorption bands at reasonable computational costs. ${ }^{31,32}$

The average $\mathrm{Chl}$ geometries from the QM/MM relaxed geometries of CaWSCP and LvWSCP show high similarity in the overall conformation and the Out Of Plane (OOP) displacement (Fig. 1c, d). The latter indicates that Chl conformation in CaWSCP is more bent than in LvWSCP, but the differences are minor when considering the root-mean squared standard deviation (RMSD). This is in contrast to the apparently flat $\mathrm{Chl}$ ring conformation in the crystal structure of LvWSCP that led Bednarczyk et al. ${ }^{19}$ to the conclusion that ring deformation is the major contribution to the spectral shift between CaWSCP and LvWSCP. Prompted by this discrepancy we found a rerefined model from the PDB-REDO database ${ }^{33}$ in which the electron densities assigned to $\mathrm{Chl}$ are better fitted with a bent macrocycle conformation. This implies that the original flat Chl conformation is poorly fitted to the electron density map (see supporting information for detailed analysis), while the simulated conformations are a better fit.

Beyond providing confidence in our simulation protocol, our findings underline the need to consider an ensemble of Chl conformations rather than a single snapshot. Calculations considering only a single conformation should be taken with caution since it might not be representative. This may be because it is a rare and extreme conformation out of a whole ensemble, or in the case of geometries extracted from a crystal structure, due to errors in a structure's refinement process resulting in a poor local fit to actual electron density maps.

The computed $\lambda_{\max }$ values for CaWSCP and LvWSCP were $2.037 \mathrm{eV}$ and $2.063 \mathrm{eV}$, respectively. The $0.026 \mathrm{eV}$ difference between these values is almost identical to the measured spectral shift between CaWSCP and LvWSCP (Fig. 2). The high accuracy in reproducing the spectral shift between CaWSCP and LVWSCP is due to error cancelation when considering the energy differences between two similar molecular systems. However, the actual positions of absorption maxima are $0.195 \mathrm{eV}$ higher than the respective measured values. A similar difference was reported between calculated and experimental gas phase absorption maxima of Chl. ${ }^{34-36}$ Since we are interested in the relative shift between the two WSCP types, this level of theory is sufficiently accurate for our purposes and we use it in order to elucidate the molecular mechanisms for tuning the Chl spectra in WSCPs. More specifically, the relative contribution of protein-induced Chl conformational changes on one hand, and Chlprotein electrostatic interactions on the other hand.

To estimate the contribution of Chl's conformational changes to the spectral shift, we calculated the excitation energies after zeroing the charges of the protein environment and keeping the Chl geometry from the QM/MM simulations (Fig. 2). The result reflects the net contribution of the $\mathrm{Chl}$ distortion to the spectral shift. For the $\mathrm{Q}_{\mathrm{y}}$ absorption band, this was found to be $0.010 \mathrm{eV}$, which is ca. a third (38\%) of the total spectral shift between the two WSCPS. This minor contribution is consistent with the minor conformational changes found in the MM simulations. 


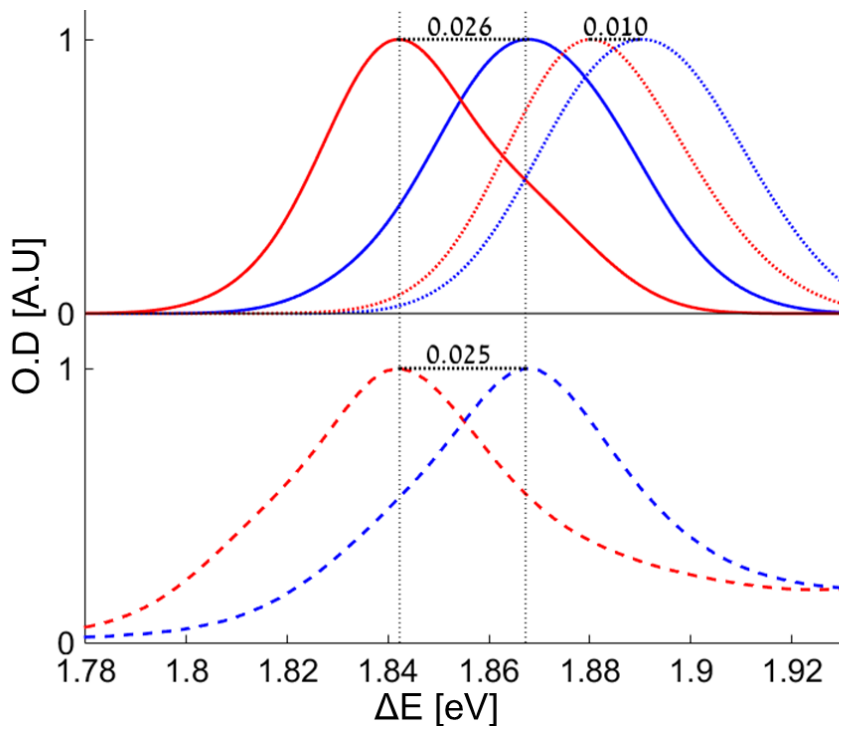

Figure 2. Effects of the $\mathrm{Chl}$ geometry and protein environment on the excitation energies. Calculated spectra of CaWSCP (red) and LvWSCP (blue) in protein with protein charges set to zero (top, dotted lines). Spectral bands calculated by considering the protein charges (top, solid lines) were shifted by $0.195 \mathrm{eV}$ in order to best match the experimental spectra (bottom, dashed lines).

Since geometry changes appear to account for about a third of the spectral shift between the two WSCP types, the rest of the shift is most likely due to a direct protein-induced electrostatic effect. The calculated contribution of Chl geometry and protein electrostatics to the excited state energy difference between LvWSCP and CaWSCP are summarized in Fig. 3.

To rationalize how the different electrostatic interactions of the $\mathrm{Chl}$ with its protein environment induce the spectral shift we inspected the changes in electron density between the ground and excited states of $\mathrm{Chl}$ in the gas phase. The difference between these electron densities (Fig. 4) reveals that electron density is shifted from rings $B$ and $D$ towards rings $A$ and $C$ upon excitation. Thus, interactions between the increased electron density and a negative charge on ring $A$ will raise the energy of the excited state more than the ground state, resulting in a blue shift. Conversely, interactions with a positive charge on ring $A$ will lower the excited state energy more than the ground state, resulting in a red shift. The opposite effect is expected on ring $B$, namely, a negative charge will result in a red shift, and a positive charge in a blue shift. A simulation of the combined effect of point charges near rings $A$ and $B$ demonstrating this concept is shown in the supporting information section S3. The use of such a point charge model to rationalize spectral tuning was reported for retinal and other chromophores ${ }^{37}$.

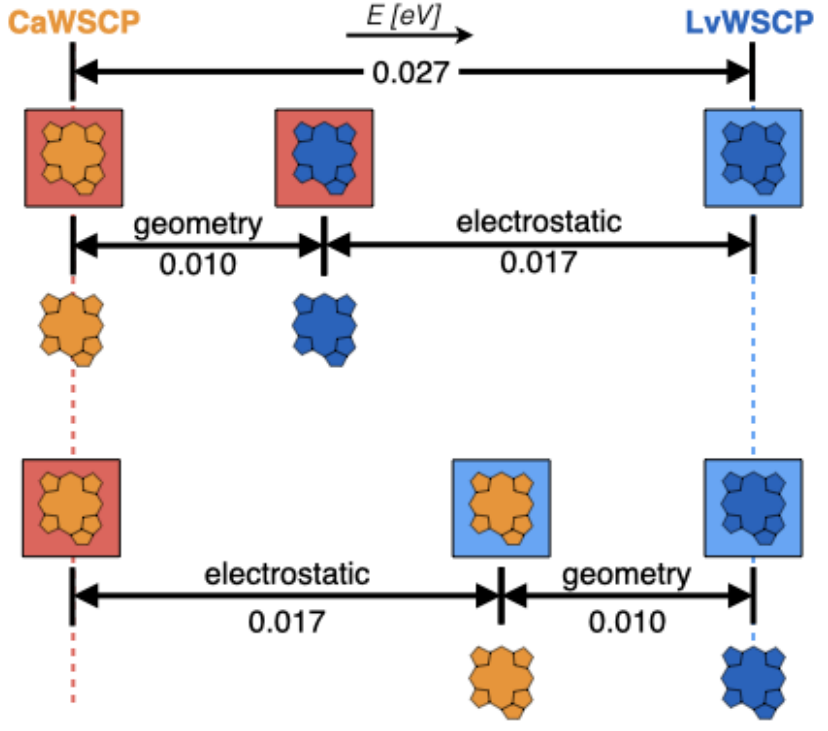

Figure 3. Relative contributions of pigment geometry and proein electrostatics to the excitation energy difference between CaWSCPand LvWSCP-Chl a complexes. Excitation energy shift due to Chl geometry changes was found to be $0.010 \mathrm{eV}$ by calculating the excitation energy of Chls within CaWSCP and LvWSCP (represented in orange, and blue, respectively) with the protein charges set to zero. The rest of the shift is due to electrostatic effects induced by the CaWSCP and LVWSCP protein environments (represented by red, and light blue rectangles, respectively).

Mapping the electrostatic potential induced by the protein environment onto the van-derWaals surface of the $\mathrm{Chl}$ reveals a more positive potential on ring $A$ in CaWSCP than in LvWSCP, and a more negative one on ring $B$ (Fig. 5). This electrostatic picture is consistent with the observed red, and blue spectral shifts. To identify which residues are responsible for the electrostatic potential differences we looked at contributions to the electrostatic potential of specific residues in the vicinity of $\mathrm{Chl}$ rings $\mathrm{A}$ and B (Fig. 5). The A34/W151 pair clearly induces a positive potential on $\mathrm{Chl}$ ring $A$ in CaWSCP, whereas the equivalent N38/W154 pair has little contribution to the electrostatic potential in this region of LvWSCP. In the vicinity of $\mathrm{Chl}$ ring $\mathrm{B}$, the nearby backbone oxygen of valine V89 in CaWSCP induces a negative electrostatic potential, whereas the backbone oxygen of the equivalent leucine L91 in LVWSCP is facing away from the ring and does not induce a negative potential. The different orientation of the backbone oxygens is caused by the different length and sequence of the PVCNEL, and LCPS loops spanning residues 88-93, and 91-94 of CaWSCP, and LvWSCP. This implies that point mutations that can change the electrostatic potential on $\mathrm{Chl}$ rings $\mathrm{A}$ and $\mathrm{B}$ may induce significant spectral shifts. 


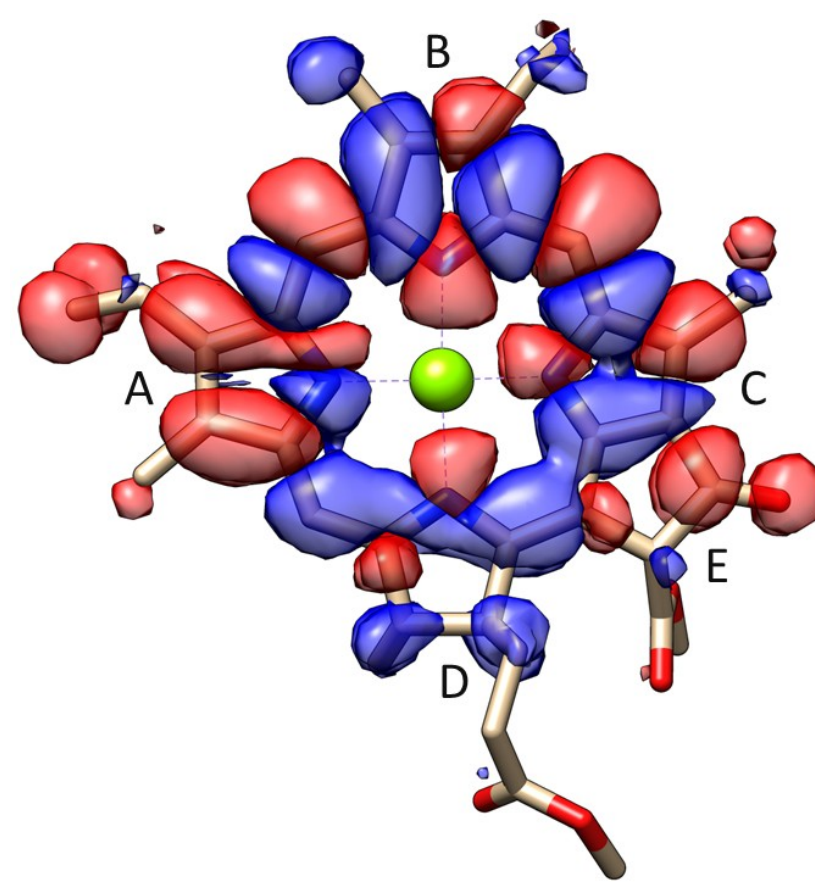

Figure 4. Electron density difference between the ground and excited state $\left(\rho_{\text {ground }}-\rho_{\text {excited }}\right)$ of the gas-phase optimized Chl. Blue and red represent positive and negative electron density difference, respectively.

Palm et al. 13,24,38 have recently targeted the same loops in exploring their effect on complex stability, and $\mathrm{Chl} a / b$ binding preferences. Their work revealed that mutating $L 91$ to $P, V, A$, or $G$ in LvWSCP has no effect on the Chl spectra, but partially or completely replacing it with the PVCNEL loop red shifts the Qy peak up to 670 $\mathrm{nm}$. While this is consistent with variations of the backbone oxygen conformation affecting the Chl spectra, when the PVCNEL of CaWSCP was replaced by LCPS the blue shift was minor. Thus, predicting spectral variations from changes in flexible regions such as the WSCP loops is not straightforward, and requires detailed structural information.
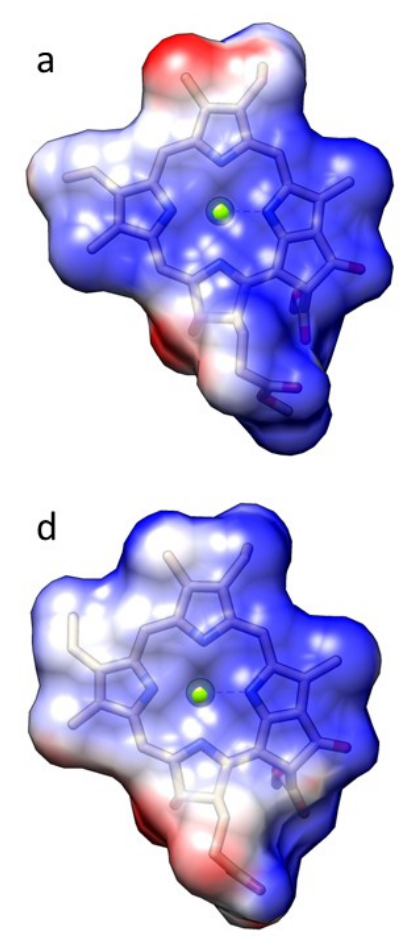
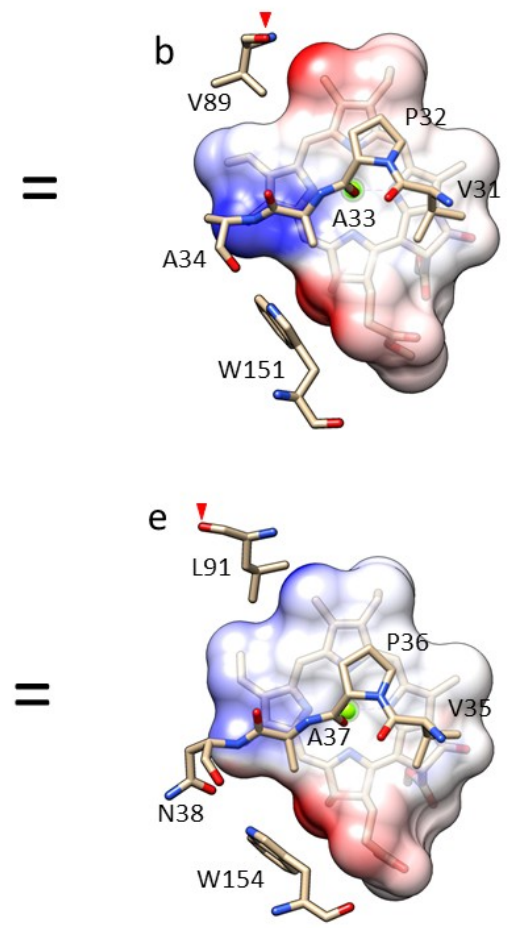
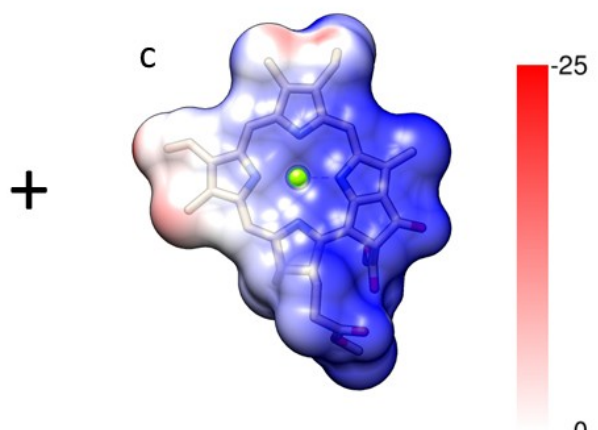

0

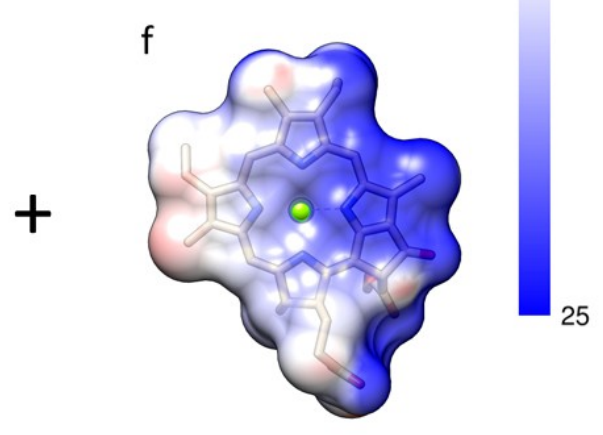

Figure 5. Electrostatic potential on the molecular surface of the Chl induced by the protein environment. Potential is presented in units of $\left[\frac{k T}{e}\right]$. a) Total electrostatic potential in CaWSCP. b) Potential induced only by the nearby residues shown in a stick representation. c) Potential induced by the rest of the CaWSCP protein. d) Total potential in LvWSCP. e) Potential induced by the nearby residues shown in a stick representation. f) Potential induced by the rest of the LvWSCP protein. The backbone oxygens of CaWSCP V89 and LvWSCP L91 are marked by a red triangle. The former is facing ring B resulting in a negative potential on the ring, whereas the latter is facing away from the ring resulting in low positive potential in the same area.

In conclusion, we have rationalized the origin of Chl absorption spectral shifts in WSCP by applying a computational protocol that combines sampling from MD simulations, QM/MM relaxation and TD-DFT calculations of excitation energies. As demonstrated here for CaWSCP- and LvWSCP-bound Chls, while variations in Chl geometry such as ring deformation may significantly contribute to the spectral band shift in specific conformations, 
when the whole ensemble is considered, electrostatic potential induced by the protein environment is the dominant effect. This is in contrast to the case of BChls in $\mathrm{LH} 2$ and $\mathrm{LH} 3$ described by De Vico et al., ${ }^{27}$ which did not consider conformational ensembles. In our case, Chl conformational changes have a smaller contribution because the conformational variations in Chls bound to CaWSCP or LvWSCP become smaller when averaged over the ensemble of conformations in each species.

The predominance of electrostatic interactions over conformational changes may have practical implications for designing novel Chl-protein complexes with prescribed spectral properties since controlling and manipulating Chl conformational variations by their protein surrounding is very difficult unless a very rigid scaffold is provided. By contrast, variations in electrostatic potential can be introduced by mutations of specific amino acid residues. Such variations can be readily implemented in protein design algorithms to provide effective tools for controlling Chl light-absorption properties by variations of their protein environment. These may be used for constructing novel light-harvesting complexes with prescribed spectral properties either by modifying natural Chl-protein complexes, or by de novo design of new Chl-binding proteins.

\section{Computational Methods}

The computational protocol is described in detail in the Supporting Information. Briefly, MD simulations were carried out using the AMBER force field ${ }^{39,40}$ starting from the crystal structures as initial geometries. Extracted geometries were subject to a QM/MM relaxation protocol, whereby the $\mathrm{QM}$ region consisted of Chl without its phytyl chain, the proline providing its backbone oxygen as an axial ligand to the Chl's central magnesium, and the alanine that follows it (P32 and A33 in CaWSCP, P36 and A37 in LvWSCP, respectively) (Fig. 1). The geometry optimization was carried out at the B3LYP $^{41} /$ Def2-SVP ${ }^{42} \mathrm{QM}$ level of theory including Grimme's dispersion correction with Becke-Johnson damping ${ }^{43}$. The QM/MM excitation energies were then calculated for each relaxed geometry using CAMB3LYP $^{44} /$ Def2-SVP level of theory. In this step, three additional residues were treated quantum mechanically, namely, A34 in CaWSCP, and N38 in LvWSCP, the respective hydrogen bonded tryptophans, W151 and W154, and another tryptophan sidechain located near $\mathrm{Chl}$ ring $B$ (W87 and W90 in CaWSCP and LvWSCP, respectively). In all calculations, both ground and excited states, the RIJCOSX ${ }^{45}$ approximation was applied. Eventually the computed spectrum was composed of a sum of 100 individual excitation energies homogeneously broadened with a Gaussian line shape with full width at half maximum of $0.025 \mathrm{eV}$.

\section{ASSOCIATED CONTENT}

This material is available free of charge via the Internet at http://pubs.acs.org." For instructions on what should be included in the Supporting Information as well as how to prepare this material for publication, refer to the journal's Instructions for Authors.

\section{AUTHOR INFORMATION}

\section{Corresponding Author}

\section{Dror Noy: drorn@migal.org.il Igor Schapiro: igor.schapiro@mail.huji.ac.il}

\section{Present Addresses}

tIf an author's address is different than the one given in the affiliation line, this information may be included here.

\section{Author Contributions}

The manuscript was written through contributions of all authors. / All authors have given approval to the final version of the manuscript. / \$These authors contributed equally. (match statement to author names with a symbol)

\section{Funding Sources}

Any funds used to support the research of the manuscript should be placed here (per journal style).

\section{Notes}

Any additional relevant notes should be placed here.

\section{ACKNOWLEDGMENT}

DN and IS acknowledge support from the Israel Ministry of Science and Technology (grant 316311). IS has received funding from the European Research Council (ERC) under the European Union's Horizon 2020 research and innovation program (grant agreement 678169 "PhotoMutant"). D.N. acknowledges financial support from the ERC consolidator grant (GA 615217), and Israel Science Foundation personal grants (GA 558/14, and GA 1338/19).

\section{ABBREVIATIONS}

Chl, chlorophyll; WSCP, water soluble chlorophyll binding protein; $\mathrm{Ca}$, cauliflower; Lv, lepidium verginicum (Virginia pepperweed); QM/MM, quantum mechanics/molecular mechanics; DFT, density functional theory; TD-DFT, time dependent - density functional theory; OOP, out of plane; VEQ, vertical excitation at equilibrium geometry.

\section{REFERENCES}


Photosystem I. Biochem. Soc. Trans. 2018, 46

(2),

Croce, R.; Van Amerongen, H. The Complex That Conquered the Land Cryo-Electron Microscopy Reveals the Structure of the Plant Photosystem II Supercomplex. Science 2017, 357 (6353), 752. https://doi.org/10.1126/science.aao4191.

Croce, R.; Van Amerongen, H. Natural Strategies for Photosynthetic Light Harvesting. Nat. Chem. Biol. 2014, 10 (7), 492-501. https://doi.org/10.1038/nchembio.1555.

(4) Kreisbeck, C.; Aspuru-Guzik, A. Efficiency of Energy Funneling in the Photosystem II Supercomplex of Higher Plants. Chem. Sci. 2016, 7 (7), 4174-4183. https://doi.org/10.1039/c5sc04296h.

(5) Neugebauer, J. Photophysical Properties of Natural Light-Harvesting Complexes Studied by Subsystem Density Functional Theory. J. Phys. Chem. B 2008, 112 (7), 2207-2217. https://doi.org/10.1021/jp709956k.

(6) König, C.; Neugebauer, J. First-Principles Calculation of Electronic Spectra of LightHarvesting Complex II. Phys. Chem. Chem. Phys. 2011, 13 (22), 10475-10490. https://doi.org/10.1039/c0cp02808h.

(7) König, C.; Neugebauer, J. Quantum Chemical Description of Absorption Properties and ExcitedState Processes in Photosynthetic Systems. ChemPhysChem 2012, 13 (2), 386-425. https://doi.org/10.1002/cphc.201100408.

(8) Suomivuori, C. M.; Winter, N. O. C.; Hättig, C.; Sundholm, D.; Kaila, V. R. I. Exploring the LightCapturing Properties of Photosynthetic Chlorophyll Clusters Using Large-Scale Correlated Calculations. J. Chem. Theory Comput. 2016, 12 (6), 2644-2651. https://doi.org/10.1021/acs.jctc.6b00237.

(9) Curutchet, C.; Mennucci, B. Quantum Chemical Studies of Light Harvesting. Chem. Rev. 2017, 117 (2), 294-343. https://doi.org/10.1021/acs.chemrev.5b00700.

(10) Cupellini, L.; Caprasecca, S.; Guido, C. A.; Müh, F.; Renger, T.; Mennucci, B. Coupling to Charge Transfer States Is the Key to Modulate the Optical Bands for Efficient Light-Harvesting in Purple Bacteria. J. Phys. Chem. Lett. 2018, acs.jpclett. 8 b03233.

https://doi.org/10.1021/acs.jpclett.8b03233.

(11) List, N. H.; Curutchet, C.; Knecht, S.; Mennucci, B.; Kongsted, J. Toward Reliable Prediction of the Energy Ladder in Multichromophoric Systems: A Benchmark Study on the FMO Light-Harvesting Complex. J. Chem. Theory Comput. 2013, 9 (11), 4928-4938. https://doi.org/10.1021/ct400560m.

(12) Jornet-Somoza, J.; Alberdi-Rodriguez, J.; Milne, B. F.; Andrade, X.; Marques, M. A. L.; Nogueira, F.; Oliveira, M. J. T.; Stewart, J. J. P.; Rubio, A. Insights into Colour-Tuning of Chlorophyll Optical Response in Green Plants. Phys. Chem. Chem. Phys. 2015, 17 (40), 26599-26606. https://doi.org/10.1039/c5cp03392f.

(13) Renger, G.; Pieper, J.; Theiss, C.; Trostmann, I.; Paulsen, H.; Renger, T.; Eichler, H. J.; Schmitt, F. J. Water Soluble Chlorophyll Binding Protein of Higher Plants: A Most Suitable Model System for Basic Analyses of Pigment-Pigment and PigmentProtein Interactions in Chlorophyll Protein Complexes. J. Plant Physiol. 2011, 168 (12), 1462-1472.

https://doi.org/10.1016/j.jplph.2010.12.005.

(14) Adolphs, J.; Maier, F.; Renger, T. WavelengthDependent Exciton-Vibrational Coupling in the Water-Soluble Chlorophyll Binding Protein Revealed by Multilevel Theory of Difference
Fluorescence Line-Narrowing. J. Phys. Chem. B 2018 122 , 8891-8899.

https://doi.org/10.1021/acs.jpcb.8b08410. Agostini, A.; Palm, D. M.; Paulsen, H.; Carbonera, D. Optically Detected Magnetic Resonance of Chlorophyll Triplet States in Water-Soluble Chlorophyll Proteins from Lepidium Virginicum: Evidence for Excitonic Interaction among the Four Pigments. J. Phys. Chem. B 2018, 122 (23), 6156-6163.

https://doi.org/10.1021/acs.jpcb.8b01906.

(16) Alster, J.; Lokstein, H.; Dostál, J.; Uchida, A.; Zigmantas, D. 2D Spectroscopy Study of WaterSoluble Chlorophyll-Binding Protein from Lepidium Virginicum. J. Phys. Chem. B 2014, 118 (13), 3524-3531. https://doi.org/10.1021/jp411174t.

(17) Fresch, E.; Meneghin, E.; Agostini, A.; Paulsen, H.; Carbonera, D.; Collini, E. How the Protein Environment Can Tune the Energy, the Coupling, and the Ultrafast Dynamics of Interacting Chlorophylls: The Example of the Water-Soluble Chlorophyll Protein. J. Phys. Chem. Lett. 2020. 11 (3), 1059-1067. https://doi.org/10.1021/acs.jpclett.9b03628.

(18) Kell, A.; Bednarczyk, D.; Acharya, K.; Chen, J.; Noy, D.; Jankowiak, R. New Insight into the Water-Soluble Chlorophyll-Binding Protein from Lepidium Virginicum. Photochem. Photobiol. 2016, 92 (3), 428-435. https://doi.org/10.1111/php.12581.

(19) Bednarczyk, D.; Dym, O.; Prabahar, V.; Peleg, Y.; Pike, D. H.; Noy, D. Fine Tuning of Chlorophyll Spectra by Protein-Induced Ring Deformation. Angew. Chemie - Int. Ed. 2016, 55 (24), 69016905. https://doi.org/10.1002/anie.201512001.

(20) Oonishi, I.; Satoh, H.; Nakagawa, A.; Itoh, N.; Uchida, A.; Mitsunaga, K.; Horigome, D. Structural Mechanism and Photoprotective Function of Water-Soluble Chlorophyll-Binding Protein. J. Biol. Chem. 2006, 282 (9), 65256531. https://doi.org/10.1074/jbc.m609458200.

(21) Prabahar, V.; Afriat-Jurnou, L.; Paluy, I.; Peleg, Y.; Noy, D. New Homologues of Brassicaceae WaterSoluble Chlorophyll Proteins Shed Light on Chlorophyll Binding, Spectral Tuning, and Molecular Evolution. FEBS J. 2020, 287 (5), 9911004. https://doi.org/10.1111/febs.15068.

(22) Agostini, A.; Meneghin, E.; Gewehr, L.; Pedron, D.; Palm, D. M.; Carbonera, D.; Paulsen, H.; Jaenicke, E.; Collini, E. How Water-Mediated Hydrogen Bonds Affect Chlorophyll a/b Selectivity in Water-Soluble Chlorophyll Protein. Sci. Rep. 2019, 9 (1), 1-10. https://doi.org/10.1038/s41598-019-54520-4.

(23) Palm, D. M.; Agostini, A.; Averesch, V.; Girr, P.; Werwie, M.; Takahashi, S.; Satoh, H.; Jaenicke, E.; Paulsen, H. Chlorophyll a/b Binding-Specificity in Water-Soluble Chlorophyll Protein. Nat. Plants 2018, 4 (11), 920-929. https://doi.org/10.1038/s41477-018-0273-z. Palm, D. M.; Agostini, A.; Pohland, A. C.; Werwie, M.; Jaenicke, E.; Paulsen, H. Stability of WaterSoluble Chlorophyll Protein (WSCP) Depends on Phytyl Conformation. ACS Omega 2019, 4 (5), 7971-7979.

https://doi.org/10.1021/acsomega.9b00054.

(25) Zucchelli, G.; Brogioli, D.; Casazza, A. P.; Garlaschi, F. M.; Jennings, R. C. Chlorophyll Ring Deformation Modulates Qy Electronic Energy in Chlorophyll-Protein Complexes and Generates Spectral Forms. Biophys. J. 2007, 93 (6), 22402254. https://doi.org/10.1529/biophysj.107.104554

(26) Senge, M. O.; MacGowan, S. A.; O'Brien, J. M. Conformational Control of Cofactors in Nature- 
the Influence of Protein-Induced Macrocycle Distortion on the Biological Function of Tetrapyrroles. Chem. Commun. 2015, 51 (96), 17031-17063.

https://doi.org/10.1039/c5cc06254c.

(27) De Vico, L.; Anda, A.; Osipov, V. A.; Madsen, A. $\varnothing$.; Hansen, T. Macrocycle Ring Deformation as the Secondary Design Principle for LightHarvesting Complexes. Proc. Natl. Acad. Sci. 2018, 115 (39), E9051-E9057. https://doi.org/10.1073/pnas.1719355115.

(28) Koolhaas, M. H. C.; van der Zwan, G.; van Grondelle, R. Local and Nonlocal Contributions to the Linear Spectroscopy of Light-Harvesting Antenna Systems. J. Phys. Chem. B 2002, 104 (18),

4489-4502.

https://doi.org/10.1021/jp9918149.

(29) Jurinovich, S.; Viani, L.; Prandi, I. G.; Renger, T.; Mennucci, B. Towards an Ab Initio Description of the Optical Spectra of Light-Harvesting Antennae: Application to the CP29 Complex of Photosystem II. Phys. Chem. Chem. Phys. 2015, 17 (22). 14405-14416. https://doi.org/10.1039/C4CP05647G.

(30) Loco, D.; Buda, F.; Lugtenburg, J.; Mennucci, B. The Dynamic Origin of Color Tuning in Proteins Revealed by a Carotenoid Pigment. J. Phys. Chem. Lett. 2018, 9 (9), 2404-2410. https://doi.org/10.1021/acs.jpclett.8b00763.

(31) Kjellgren, E. R.; Haugaard Olsen, J. M.; Kongsted, J. Importance of Accurate Structures for Quantum Chemistry Embedding Methods: Which Strategy Is Better? J. Chem. Theory Comput. 2018, 14 (8), 4309-4319. https://doi.org/10.1021/acs.jctc.8b00202.

(32) Martina De Vetta, Omar Baig, Dorika Steen, Juan J. Nogueira, L. G. Assessing Configurational Sampling in the Quantum Mechanics/Molecular Mechanics Calculation of Temoporfin Absorption Spectrum and Triplet Density of States. Molecules 2018, $23 \quad$ (11), 2932. https://doi.org/10.3390/molecules23112932.

(33) Joosten, R. P.; te Beek, T. A. H.; Krieger, E.; Hekkelman, M. L.; Hooft, R. W. W.; Schneider, R.; Sander, C.; Vriend, G. A Series of $\{$ PDB $\}$ Related Databases for Everyday Needs. Nucleic Acids Res. 2010, 39 (Database), D411--D419. https://doi.org/10.1093/nar/gkq1105.

(34) Cai, Z. L.; Crossley, M. J.; Reimers, J. R.; Kobayashi, R.; Amos, R. D. Density Functional Theory for Charge Transfer: The Nature of the NBands of Porphyrins and Chlorophylls Revealed through CAM-B3LYP, CASPT2, and SAC-CI Calculations. J. Phys. Chem. B 2006, 110 (31), 15624-15632.

https://doi.org/10.1021/jp063376t.

(35) Milne, B. F.; Toker, Y.; Rubio, A.; Nielsen, S. B.
Unraveling the Intrinsic Color of Chlorophyll. Angew. Chemie - Int. Ed. 2015, 54 (7), 21702173. https://doi.org/10.1002/anie.201410899.

(36) Parusel, A. B. J.; Grimme, S. A Theoretical Study of the Excited States of Chlorophyll $a$ and Pheophytin A . J. Phys. Chem. B 2002, 104 (22), 5395-5398. https://doi.org/10.1021/jp000346w.

(37) Orozco-Gonzalez, Y.; Kabir, M. P.; Gozem, S. Electrostatic Spectral Tuning Maps for Biological Chromophores. J. Phys. Chem. B 2019, 123 (23), 4813-4824.

https://doi.org/10.1021/acs.jpcb.9b00489.

(38) Palm, D. M.; Agostini, A.; Avaresch, V.; Girr, P.; Werwie, M.; Takahashi, S.; Satoh, H.; Jaenicke, E.; Paulsen, $\mathrm{H}$. Chlorophyll a/b Binding-Specificity in Water-Soluble Chlorophyll Protein. Nat. Plants 2018, 4 (November). https://doi.org/10.1038/s41477-018-0273-z.

(39) Simmerling, C.; Cheatham, T. E.; Wang, B.; Case, D. A.; Merz, K. M.; Darden, T.; Gohlke, H.; Onufriev, A.; Woods, R. J.; Luo, R. The Amber Biomolecular Simulation Programs. J. Comput. Chem. 2005, 26 (16), 1668-1688. https://doi.org/10.1002/jcc.20290.

(40) Maier, J. A.; Simmerling, C.; Wickstrom, L.; Hauser, K. E.; Martinez, C.; Kasavajhala, K. Ff14SB: Improving the Accuracy of Protein Side Chain and Backbone Parameters from Ff99SB. J. Chem. Theory Comput. 2015, 11 (8), 36963713. https://doi.org/10.1021/acs.jctc.5b00255.

(41) Becke, A. D. Density-functional Thermochemistry. III. The Role of Exact Exchange. J. Chem. Phys. 1993, 98 (7), 56485652. https://doi.org/10.1063/1.464913.

(42) Weigend, F.; Ahlrichs, R. Balanced Basis Sets of Split Valence, Triple Zeta Valence and Quadruple Zeta Valence Quality for $\mathrm{H}$ to Rn: Design and Assessment of Accuracy. Phys. Chem. Chem. Phys. 2005, 7 (18), 3297-3305. https://doi.org/10.1039/B508541A.

(43) Grimme, S.; Ehrlich, S.; Goerigk, L. Effect of the Damping Function in Dispersion Corrected Density Functional Theory. J. Comput. Chem. 2011, 32 (7), 1456-1465. https://doi.org/10.1002/jcc.

(44) Yanai, T.; Tew, D. P.; Handy, N. C. A New Hybrid Exchange-Correlation Functional Using the Coulomb-Attenuating Method (CAM-B3LYP). Chem. Phys. Lett. 2004, 393 (1-3), 51-57. https://doi.org/10.1016/j.cplett.2004.06.011.

(45) Neese, F.; Wennmohs, F.; Hansen, A.; Becker, U. Efficient, Approximate and Parallel Hartree-Fock and Hybrid DFT Calculations. A "chain-ofSpheres" Algorithm for the Hartree-Fock Exchange. Chem. Phys. 2009, 356 (1-3), 98109.

https://doi.org/10.1016/j.chemphys.2008.10.036. 


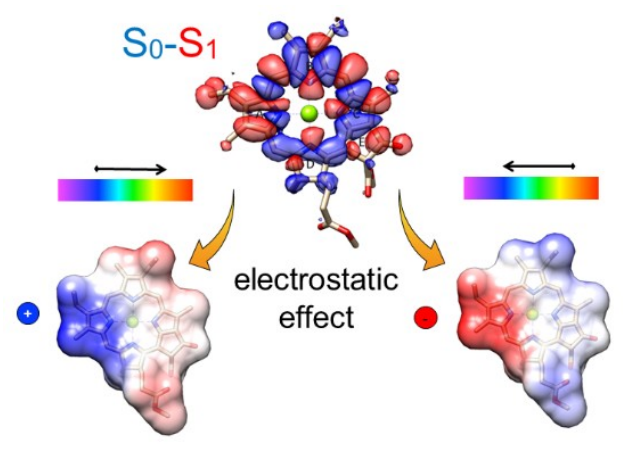

